\title{
NINA BIGOT
}

\section{Université de Lille}

\section{La Nuit Épuisée : \\ poïétique d'une insomnie collective}

$\left[\begin{array}{c}\text { a Nuit Épuisée, ouvrage à l'initiative d'Antoine } \\ \text { d'Agata }{ }^{1} \text { et Léa Bismuth }{ }^{2} \text {, repose sur une }\end{array}\right.$ expérience d'écriture : celle d'une nuit blanche collective à la Friche de la Belle de Mai, au Cabaret Aléatoire de Marseille.

C'est la nuit du 17 au 18 février 2018 que Philippe Azoury, Mehdi Belhaj Kacem, Léa Bismuth, Jean-Baptiste Del Amo, Mathilde Girard, Yannick Haenel, Bruno le Dantec, Emmanuel Loi, Bernard Marcadé, Guillaume de Sardes, John Jefferson Selve et Antoine d'Agata ont épuisée, au cours d'une insomnie qui a engendré cet ouvrage

\footnotetext{
1 Antoine d'Agata est connu en tant que photographe français contemporain. Ses ouvrages photolittéraires, souvent issus de collaborations avec des auteurs (Bruno le Dantec, Rafael Garido, etc), sont à la fois viatique et autobiographiques.

2 Léa Bismuth est une auteure, critique d'art, commissaire d'exposition et enseignante française. Ses travaux de recherche portaient sur la pensée de Georges Bataille et de Pierre Klossowski, et, dans le domaine de l'art contemporain, sur la photobiographie.
} 
composé de douze leporelli sérigraphiés. Comme beaucoup d'ouvrages agatiens - Ice et Agonie, écrit avec Rafael Garido, Codex, qui mêle les textes d'Agata à ceux de Bruno le Dantec, Tania Bohorquez et Nicolas Arraitz ou encore Atlas, collection de paroles de prostituées - La Nuit Épuisée repose sur une polyphonie auctoriale. Dans La Nuit Épuisée, à chaque auteur correspond un texte; à chaque texte correspond une expérience personnelle de cette insomnie collective; à chaque expérience correspond un leporello dont le titre, constitué d'un mot, est une porte d'entrée au cœur de cette nuit blanche. Leurs textes se mêlent aux photographies d'Antoine d'Agata, qui bénéficient, grâce à la sérigraphie, d'un nouveau traitement graphique.

Cette nuit blanche se déroule dans une boîte de nuit qui diffuse, sur ses écrans, des extraits de White Noise, film d'Agata composé de «paroles de femmes de Tokyo à Bogota en passant par Phnom Penh, Oslo et Kiev » ${ }^{3}$; film qui se conjugue aux musiques de La Bande Adhésive et Métaphore Collectif.

3 Auteur inconnu, « Dérive », [dans :] A. d'Agata, La Nuit Épuisée, Marseille, Friche la Belle de Mai / André Frère Éditions, 2018 (ouvrage non numéroté). 
Il ne s'agit donc pas seulement pour ces auteurs de ne pas dormir, mais de ne pas dormir tout en étant au cœur des rituels urbains de la nuit, de telle sorte qu'aucun d'eux ne puisse échapper à l'influence de cette nuit ; contexte de création analogue à la poïétique d'Agata, dont les ouvrages sont issus de ses errances nocturnes.

Que nous donne à lire cette polyphonie nocturne ? Discerne-t-on, au sein de cette prolifération de voix, une trame commune, porteuse des marques laissées par la nuit ? C'est en nous plongeant dans La Nuit Épuisée que nous apercevrons, au sein de cette multiplicité de voix, une poïétique de l'insomnie. C'est d'abord à travers la plasticité de cet ouvrage, qui propose au lecteur une expérience perceptive analogue à celle vécue par les auteurs, qu'il sera possible de voir "ce qu'on ne voit que dans la nuit " 4 blanche. Dans la mesure où l'objet de la poïétique est « le rapport dynamique qui [unit l'artiste] à son œuvre pendant qu'il est aux prises avec elle ${ }^{5}$, notre intérêt se portera ensuite sur la nuit en tant qu'espace géographico-corporel parcouru au cours de l'insomnie, puis sur les caractéristiques

4Y. Haenel, «Ivresse », [dans :] A. d'Agata, La Nuit Épuisée, op. cit. 5R. Passeron, Pour une philosophie de la création, Paris, Klincksieck, 1989, p. 16. 
de la temporalité nocturne, afin de déduire du contexte de création de l'œuvre les spécificités de l'écriture nocturne.

Plasticité de la photolittérature nocturne

La Nuit Épuisée, par sa forme, impose un nouveau rapport au livre, aux textes, à la lecture. Composé de leporelli, qui sont autant d'espaces à déplier et à parcourir comme autant de portes à ouvrir, il fait écho à l'œuvre ouverte conceptualisée par Umberto Eco. Le corps s'implique dans la lecture : I'ouvrage se manipule, tout comme la constellation de ses leporelli qui se dispersent, se mêlent et se répondent.

L'expérience d'écriture à l'origine de cet ouvrage - décrit comme " [une] fosse commune où ça cause " ${ }^{6}$ - est plurielle ; sa polyphonie se matérialise à travers cette conception du livre, où chaque leporello revendique sa singularité, hors de tout ordonnancement. Le lecteur est libre, exempt de tout influence ; aucune trajectoire n'est tracée, pour lui, en amont, dans cet ouvrage sans début ni fin explicites.

"Bout à bout, les images reconstruisent un puzzle aléatoire qui repousse les limites de l'explicite et figure, sans la figer ou la simplifier, une

6E. Loi, «Absence », [dans :] A. d'Agata, La Nuit Épuisée, op. cit. 
expérience ${ }^{7}$ : cette citation issue du Manifeste d'Agata illustre le fonctionnement de La Nuit Épuisée, où les leporelli sont autant de pièces d'un « puzzle aléatoire » (locution oxymorique qui souligne les infinies possibilités d'association de ces leporelli). Pour Agata, la poïétique d'une œuvre tout comme sa réception doivent être des expériences, et toutes deux doivent se répondre. L'assemblage de feuillets à la fois interdépendants (puisque faisant partie du même ouvrage) et autonomes (puisque n'étant soumis à aucun ordre de lecture) propose une expérience de lecture inédite. Les mots se déploient alors sur une toute nouvelle scène discursive - c'est-à-dire l'espace dans lequel s'inscrit le discours - où la pluralité des leporelli matérialise la polyphonie de cet ouvrage. Libre au lecteur d'aller de l'un à l'autre, comme s'il choisissait à quelle conversation nocturne il souhaitait se greffer.

La présence d'Agata s'ancre dans les photographies sérigraphiées qui coexistent avec les textes des différents auteurs ; à la nuit textuelle s'ajoute alors une nuit visuelle, elle aussi porteuse des marques de cette nuit blanche.

7 A. d'Agata, « Séquences », [dans :] Manifeste, Paris, Le Bleu du ciel, 2005, p. 13. 
Ce qui danse, ce n'est pas nous, mais l'intériorité elle-même de la nuit. La parole ne cesse d'y jaillir, et de voir ce qu'on ne voit que dans la nuit. Oui, la parole voit - elle « voit des images ", comme dit Antoine d'Agata, elle fait attention aux flammes, aux éclairs, à la foudre qui traversent les corps. ${ }^{8}$

À l'acte de parler s'amalgame l'acte de voir, amalgame qui constitue l'épine dorsale des ouvrages agatiens. Les différents leporelli matérialisent cette parole nocturne qui « voit des images » par le biais de leur trichromatisme commun : rouge, blanc, noir.

Ce trichromatisme de la nuit blanche porte les marques des «flammes », des « éclairs » et de «la foudre » caractéristiques de cette parole qui jaillit au cœur de la nuit.

La présence du noir n'est pas surprenante ; teinte nocturne par excellence, elle matérialise l'absence totale de luminosité caractéristique de la nuit. Sur le mode de la camera obscura, du noir jaillissent paroles et photographies. La boîte de nuit, d'où émergent les différents textes, est d'ailleurs assimilable à cette boîte qu'est la chambre noire, dont les images surgissent de l'obscurité. L'omniprésence de la noirceur indique la filiation de cet ouvrage avec la nuit qu'il représente et où il est né.

8Y. Haenel, « Ivresse », op. cit. 
La coïncidence contradictoire de l'obscurité du noir avec la forte luminosité du blanc crée un effet de contraste qui frappe l'œil à la façon de la foudre. L'image contrastée est brutale : ses contours sont tranchés. Ce contraste illustre le caractère d'apparence antinomique de la locution " nuit blanche », où l'obscurité nocturne coïncide avec la forte luminosité diurne. Le blanc fait écho au titre du film White Noise, projeté dans la boîte de nuit durant cette expérience d'écriture, titre explicité dans le leporello « Dérive » :

\section{WHITE NOISE}

Le bruit blanc, dont le nom se réfère à la " lumière blanche » qui est mélange équilibré de couleurs, est composé de toutes les fréquences audibles, chaque fréquence ayant la même énergie.

L'exposition constante à un bruit blanc de niveau élevé est une des formes de la torture par privation sensorielle. ${ }^{9}$

La Nuit Épuisée nous livre ici sa propre définition de la locution «nuit blanche ».

Qu'est-ce qui est blanc, dans la nuit blanche? Pourquoi parler de nuit blanche? Si la locution " nuit noire » insiste sur la profondeur " pure, sans plans, sans surfaces, sans distance » ${ }^{10}$ de

9 Auteur inconnu, « Dérive », op.cit.

10 M. Merleau-Ponty, Phénoménologie de la perception, Paris, Gallimard, 1945, p. 328. 
la noirceur nocturne, la blancheur de la « nuit blanche » consiste elle aussi en une épreuve pour nos sens, mais elle instaure également un rapport particulier à l'énergie qui circule en son sein. La « nuit blanche » est bel et bien « nuit épuisée ». Le corps, déjà fatigué par l'agitation du jour, s'y épuise davantage. La nuit blanche concentre, mélange et emmêle différents flux d'énergies; il n'est alors guère surprenant que la boîte de nuit - espace où divers corps se retrouvent pour danser, se mêler - en soit le lieu de prédilection. "Ce qui danse, ce n'est pas nous, mais l'intériorité elle-même de la nuit »: une intériorité blanche, qui porte la couleur de l'épreuve sensorielle épuisante d'une nuit marquée par le sceau de l'insomnie, épreuve dont les corps ressortent « épuisés, inépuisables, assoiffés ${ }^{11}$.

À l'alliage contrasté du noir et du blanc s'ajoute une dernière couleur : le rouge. Cette couleur est caractérisée par sa puissance : «Avec le rouge, on est dans l'hypersensibilisation, dans l'excès, dans I'"ubris", dans la démesure. Démesure de l'affect et démesure du geste. Le champ visuel induit une perception et une réaction excessive, affirme Merleau-Ponty à la suite de Goldstein. On

11 Y. Haenel, « Ivresse », op. cit. 
est dans l'excès parce qu'on est au plus haut point de la tension, point limite où l'appareil psychique (psychomoteur), surchargé, décharge, ou plutôt explose. Le rouge monte (comme la colère) et jaillit (comme le sang). [...] "Le rouge s'enfonce dans l'œil", dit Goethe » ${ }^{12}$.

Le rouge est la couleur de l'énergie en tant que surcharge, décharge, explosion. L'ouvrage gagne en force, tandis que la puissance du contraste noir-blanc se mêle à la violence du rouge, couleur qui marque l'œil et qui s'y enfonce. Le rouge brutal choisit pour cet ouvrage blesse volontairement la vision; voir devient physique, quand la couleur, violente, virulente, pique la rétine, gênant parfois la lecture comme le noir peut gêner l'œil qui déchiffre le nocturne. Le rouge est excès tout comme la nuit blanche est le moment des excès; elle monte et jaillit dans l'organisme à la façon de la drogue, de l'orgasme ou la musique qui «monte » et « rythme [le] sang » ${ }^{13}$. À l'instar du sang, le rouge est une couleur à la fois vitale et mortifère. Elle réchauffe l'œil et le frappe simultanément, mêlant le pouvoir des flammes et de l'éclair.

12C. Lyotard, C. Masson, «La peinture ambivalente », [dans :] G. Gassiot-Talabot et al., Figurations : 1960/1973, Paris, UGE, 1973, p. 270-271.

13 Y. Haenel, « Ivresse », op. cit. 
"Le jour appartient au pouvoir, la nuit à la puissance ${ }^{14}$; la puissance nocturne est restituée, dans La Nuit Épuisée, par le trichromatisme noirblanc-rouge, qui mêle le tranchant du contraste entre le noir et le blanc à la violence explosive du rouge.

La nuit coïncidant avec une baisse de la luminosité, la plasticité de cette photolittérature nocturne joue avec les limites de la vision, qui se fatigue et s'abîme en scrutant l'obscur. Les contours, contrastés ou tracés avec les teintes violentes du rouge, offrent une première épreuve pour l'œil. II faut s'y confronter, tout comme le corps se confronte à la nuit blanche. Si « la nuit [...] n'a pas d'ombre » ${ }^{15}$, c'est parce que l'obscurité s'y étend, rongeant l'espace, privant peu à peu la vue de son empire. Ce " qu'on ne voit que dans la nuit " ${ }^{16}$ est donc, d'abord, une épreuve pour le regard, pour qui s'acclimater à l'obscurité nécessite également s'en remettre à elle. Les corps épuisés par la nuit sont des corps qui dansent, pas des corps qui regardent. La vision bat en brèche, dans cette nuit qui se vit de façon musculaire, nerveuse, épidermique.

14L. Bismuth, «Action », [dans :] A. d'Agata, La Nuit Épuisée, op. cit. 15 Y. Haenel, « Ivresse », op. cit.

$16 \mathrm{lbidem}$. 
La photographie agatienne repose sur le flou, en tant qu'il traduit les mouvements du corps. En glissant de la photographie à la sérigraphie, les contours flous se dessinent autrement : soit par le tranchant du contraste, soit par l'indistinction de ces images en gros plan, dont le grain épais fait fusionner le fond et la forme qui s'en détache. L'image se passe de perspective, à la façon de la nuit dont la profondeur est "sans plans, sans surfaces, sans distance $» 17$.

Par le biais de la sérigraphie, la plasticité de $L a$ Nuit Épuisée matérialise la spatialité de la nuit : elle permet au lecteur de parcourir la nuit en parcourant l'ouvrage, l'inscrivant dans le sillage des auteurs noctambules. L'expérience esthétique de lecture tend alors à se superposer à l'expérience poïétique d'écriture.

La nuit comme espace géographico-corporel

Quiconque passe une nuit blanche se tient « dans la nuit » ${ }^{18}$; il participe à une expérience sensorielle où son sens de prédilection, la vue, est mise à mal, et où les autres sens " sont en permanence susceptibles de se contredire les

17 M. Merleau-Ponty, Phénoménologie de la perception, op. cit., p. 328.

$18 \mathrm{M}$. Foessel, La Nuit. Vivre sans témoin, Paris, Autrement, 2017, p. 49. 
uns les autres $"{ }^{19}$. Au cours de cette nuit blanche collective, la vue (la projection de White Noise) se mêle à l'ouïe (de La Bande Adhésive et Métaphore Collectif), tandis que les corps dansent, que les bouches parlent, que les doigts écrivent. La nuit est alors sensorielle, mais aussi musculaire quand elle épuise celui qui la vit - et organique, quand elle coïncide avec une consommation de «bizarres substances » ${ }^{20}$ qui altèrent perception, compréhension, façon de sentir et de se sentir dans son corps.

Si la nuit blanche est corporelle, c'est parce qu'elle réclame une implication totale du corps dans l'expérience qu'elle lui propose, d'où le rituel urbain qui lui correspond : une nuit blanche passée dans une boîte de nuit où le corps se laisse rythmer par l'espace qu'il habite.

La nuit, pour Agata qui estime que «les corps font espace », qu'ils " incorporent et constituent un espace » ${ }^{21}$, se conçoit alors en tant qu'espace, à la fois urbain et corporel. À chaque leporello correspond une incorporation de cette nuit urbaine.

La boîte de nuit est au cœur des rituels de la nuit ; nocturne par essence, elle creuse, dans

19 lbidem, p. 60.

20Y. Haenel, « Ivresse », op. cit.

21 A. D'Agata, R. Garido, Agonie, Actes Sud, 2009, p. 22. 
l'obscurité de la ville, un espace dédié à la fête ; espace vers lequel convergent noctambules et insomniaques soucieux de se réunir. Elle sépare ceux qui dorment de ceux qui veillent. Cette boîte contient l'envers du jour : sans lumières franches ni horaires fixes, elle est l'espace "des dépenses sociales improductives " 22 opposées aux logiques de production et d'acquisition qui régulent les activités diurnes. Si la poïétique de La Nuit Épuisée fait écho à la dépense bataillienne, on trouve chez Guy Debord et Gilles Deleuze deux conceptions de l'espace qui illustrent son rôle dans la poïétique de la nuit blanche.

La façon dont Agata, et les auteurs de cet ouvrage collectif qui s'inscrivent dans son sillage, approche l'espace fait écho à la psychogéographie conceptualisée par Guy Debord, à savoir «l'étude des effets précis du milieu géographique, consciemment aménagé ou non, sur les comportements affectifs des individus » ${ }^{23}$. La Nuit Épuisée, née de l'effet de la nuit et de sa boîte sur chacun des auteurs, est un ouvrage psychogéographique

22G. Bataille, La Part Maudite précédée de La Notion de dépense, Paris, Minuit, 1967, p. 36.

23G. Debord, "Définitions », [dans :] Internationale Situationniste, Juin 1958, $n^{\circ} 1$, p. 13. Disponible en ligne: http://hipermedula.org/wp-content/uploads/2013/09/Internationale_situationniste_1.pdf. On trouve dans les livres d'Agata des récits des effets des lieux - et en particulier les bas-fonds sur les êtres, et en particulier sur lui-même. 
nocturne, dont chaque leporello est un échantillon. Expérimenter la nuit, c'est expérimenter le nocturne en tant qu'espace. Dans la boîte de nuit, le corps s'implique dans l'espace qui agit sur lui, au point d'entendre, au fond de son propre corps, ses « os qui chantent " ${ }^{24}$ comme pour répondre à la musique qui se répand. Ce « milieu géographique » qu'est la boîte de nuit impacte en effet les « comportements affectifs » des insomniaques:

Je le sais désormais, ce que je cherchais là, c'était l'incarnation, la chorégraphie muette de la nuit devenue geste, les corps qui tombent et qui se rattrapent, qui se mordent et qui rient, qui perdent haleine, qui s'engouffrent dans les paysages inconnus. [...] C'est une façon de vivre, une forme de vie qui féconde, ouvre le champ de joie, de puissance et de risque, tout à la fois. ${ }^{25}$

Au cœur de la boîte de nuit, les mouvements des corps nocturnes ne sont pas ceux des corps diurnes : la nuit agite et exalte le corps dans lequel elle s'incarne. Dans cet espace, la « nuit devenue geste » est protégée de toute temporalité synonyme de limite ; les corps rêvent (en vain) d'une nuit sans fin et d'un corps sans fatigue:

je voudrais vivre dans cette nuit qui tranche mon angoisse (qui me jette vers les autres), je voudrais que mes bras s'ouvrent sans s'arrêter ; j'approche mes lèvres de celles d'une femme: je voudrais embrasser sans fin. ${ }^{26}$

24 Y. Haenel, « Ivresse », op. cit.

25 L. Bismuth, «Action », [dans :] A. d'Agata, La Nuit Épuisée, op. cit. 26Y. Haenel, « Ivresse », op. cit. 
La dimension affective de cette expérience est également tangible dans la cartographie affective deleuzienne, où " [l]es cartes ne doivent pas seulement se comprendre en extension, par rapport à un espace constitué de trajets. II y a aussi des cartes d'intensité, de densité, qui concernent ce qui remplit l'espace, ce qui sous-tend le trajet $\gg{ }^{27}$. L'espace est, chez Deleuze et Agata, autant un " paysage étalé dans un champ perceptif » qu'un « champ énergétique traversé par des différences de potentiel, des gradients et des seuils, des hausses et des chutes d'intensité ${ }^{28}$. Or, cette nuit épuisée par chacun des auteurs est traversée de « bandes d'intensité », de « variations intensives » ${ }^{29}$, comme si l'espace nocturne était propice aux débordements, à la dépense : "sueur froide » 30 suite à la prise de GHB ; "sacrifices de forces » dans la dépense et danse mêlée à la «transe ${ }^{31}$; «fureur ${ }^{32}$; intensité de «l'extase orgastique ${ }^{33}$.

27 G. Deleuze, Critique et Clinique, Paris, Minuit, 1993, p. 192. 28 G. Sibertin-Blanc, "Cartographie et territoire. La spatialité géographique comme analyseur des formes de subjectivité selon Gilles Deleuze », [dans :] L'Espace Géographique, 2012, $n^{\circ} 2010 / 3$, t. 39, p. 225-238.

29 lbidem.

30 P. Azoury, «Chute», [dans :] A. d'Agata, La Nuit Épuisée, op. cit. 31 Y. Haenel, « Ivresse », op. cit.

32 Auteur inconnu, « Dérive », op. cit.

33 M. Belhaj Kacem, « Instinct », [dans :] A. d'Agata, La Nuit Épuisée, op. cit. 
Chaque auteur entretient donc un rapport intensif à la nuit blanche, mais également un rapport affectif illustré par la personnification de la nuit ; nuit « inconsolable » à l'apparence d'une «femme qui pleure » ${ }^{34}$; nuit incarnée par les prostituées de White Noise, "les sacrifiées et les seules saintes d'esprit » ${ }^{35}$. La figure de la prostituée, inhérente au travail d'Agata, traverse ainsi divers leporelli; plus qu'un emblème de la nuit, elle en devient l'incarnation. N'existant que dans l'espace nocturne, dont l'obscurité la protège et la cache, elle en est autant le fantôme que la reine.

La nuit n'est pas qu'un moment; elle crée son propre espace tandis qu'elle le plonge dans l'obscurité ; elle invoque et éveille son propre peuple au moment où elle tombe sur la ville. Si l'ouvrage reproduit plastiquement l'aspect de la nuit, il propose également la traversée de la nuit en tant qu'espace psychogéographique et affectif. L'auteur y expérimente un langage né de la dépense, "création au moyen de la perte ${ }^{36}$ mais également de l'épuisement de la nuit ainsi que des organismes qui l'ont incorporée.

34 M. Girard, « Manque », [dans :] A. d'Agata, La Nuit Épuisée, op. cit. 35J. Jefferson Selve, "Sacrifice », [dans :] A. d'Agata, La Nuit Épuisée, op. cit..

36 G. Bataille, La Part Maudite précédée de La Notion de dépense, op. cit., p. 31. 
Temporalité subversive de la nuit : paroxysme et oxymore

À la nuit correspond un régime de visibilité, un espace, mais également une temporalité ; temporalité que l'on peut qualifier de sacré, au double sens étymologique du terme : sacer, "ce qui ne peut être touché sans être souillé, ou sans souiller ${ }^{37}$. Fondamentalement oxymorique, simultanément maudite et sacrée, côtoyant à la fois le divin et le mal, elle annihile les classifications diurnes qui identifient et hiérarchisent ce qui relève du bien et du mal. C'est alors de la nuit que peut naître le récit du leporello "Désir », récit de sexe et de meurtre; c'est elle qui inspire les réflexions sur l'orgasme, l'animalité, la sexualité $d^{\prime}$ " Instinct » c'est dans la nuit que les prostituées de White Noise sont des «sacrifiées » et des "saintes ${ }^{38}$. Dans ces récits nocturnes s'opère un renversement des valeurs diurnes.

La temporalité de la nuit s'avère subversive : elle «bouleverse l'ordre établi » ${ }^{39}$, et notamment parce qu'elle est un événement perceptif transgressif. Si

37 Définition du mot « sacré » par le CNRTL. Disponible en ligne : https://www.cnrtl.fr/definition/sacr\%C3\%A9.

$38 \mathrm{~J}$. Jefferson Selve, «Sacrifice », op. cit.

39 Définition du verbe subvertir par le CNRTL. Disponible en ligne : https://www.cnrtl.fr/definition/subvertir. 
le « dérèglement nocturne des sens ${ }^{40}$ repose, nous l'avons vu, sur une expérience sensorielle où la vue est mise à mal, il correspond également au temps paroxystique de l'ivresse et de la " défonce » ${ }^{41}$ qui coïncide avec le temps de la nuit. Le rythme de "Chute » est celui du GHB, et dans " Ivresse » apparaissent de « courtes lignes blanches étalées pour faire battre les cœurs plus vite ${ }^{42}$. L'ivresse, « exaltation des facultés mentales ${ }^{43}$, fait écho à la «transe » ${ }^{44}$, à l'« euphorie » ${ }^{45}$, à I' " extase » ${ }^{46}$; autant d'états qui débordent le sujet en le hissant vers une acmé, ou qui le frappent comme le ferait la foudre au cœur de cette " nuit saturée de stridences ${ }^{47}$. L'ivresse est chevillée au corps de cette poïétique de l'insomnie. Elle « tend vers cette fluidité qui fait coïncider le temps et le corps $»{ }^{48}$, permettant à l'auteur d'ancrer son écriture dans

40 M. Foessel, La Nuit. Vivre sans témoin, op. cit., p. 60.

$41 \mathrm{J.-B}$. Del Amo, «Brûlure », [dans :] A. d'Agata, La Nuit Épuisée, op. cit.

42 Y. Haenel, « Ivresse », op. cit.

43 Définition du mot « ivresse » par le CNRTL. Disponible en ligne: https://www.cnrtl.fr/definition/ivresse.

44 Le terme revient dans «Ivresse » et « Action » (respectivement de Y. Haenel et L. Bismuth).

45 P. Azoury, "Chute », op. cit.

$46 \mathrm{M}$. Belhaj Kacem, «Instinct », [dans :] A. d'Agata, La Nuit Épuisée, op. cit.

47 L. Bismuth, «Action », op. cit.

48Y. Haenel, « Ivresse », op. cit. 
l'expérience, afin qu'à la " brûlure de l'instant » 49 correspondent des « paroles incandescentes ${ }^{50}$.

L'ivresse perceptible dans chaque leporello n'est pas uniquement le fait d'une substance ingérée. Dans la boîte de nuit, lieu où l'on danse, le rythme gouverne les corps, au point que «le pouls se règle sur la musique ${ }^{51}$. L'analyse par Pierre Savaunet de la musique dans la philosophie nietzschéenne met en avant le caractère ambivalent de la «puissance mystérieuse du rythme » qui «prend une double forme, aliénante ou libératrice : celle d'une contrainte et celle d'un plaisir - plaisir de la contrainte et contrainte du plaisir -, celle d'un envoûtement passif et celle d'un consentement actif ${ }^{52}$. L'expérience du rythme est l'expérience corporelle d'un oxymore : le corps, contraint par le rythme qui s'empare de lui, fait (paradoxalement) l'expérience d'une liberté puissante. Il est également l'expérience d'un état analogue à l'ivresse et à la transe quand il « abolit la subjectivité jusqu'au plus total oubli de soi ${ }^{53}$.

49 M. Girard, « Manque », op. cit.

50 J. Jefferson Selve, «Sacrifice », op. cit.

$51 \mathrm{P}$. Savaunet, « Nietzsche, philosophe-musicien de l'éternel retour », [dans :] Archives de Philosophie , 2001, n²001/2, t. 64,

p. 343-360.

52 lbidem.

$53 \mathrm{lbidem}$. 
L'existence tout comme l'identité d'une boîte de nuit dépend du rythme qu'elle renferme, rythme que s'injectent les corps qui y dansent, en quête d'une expérience corporelle à la fois antinomique (entre envoûtement et consentement, libération et aliénation) et paroxystique, quand le rythme envahit le corps jusqu'à le mener vers la transe. Le pouls de la nuit blanche est celui d'un corps épuisé par son expérience de l'intensité.

La « mystique laïque de l'intensité sexuelle ou stupéfiante $\gg{ }^{54}$ qui se déploie dans la nuit se répand dans ces textes nocturnes. Si l'ivresse et la « défonce » sont des transgressions de la perception diurne (caractérisée par le calcul, la prévoyance et la classification ${ }^{55}$ ), la sexualité, associée à l'obscène, s'inscrit dans leur sillage. "Désir », " Manque », «Instinct » ancrent la sexualité, de l'ordre de l'intime et du secret, dans la nuit; ce qui ne peut pas se montrer se déroule à l'abri des regards, au moment où l'obscurité peut servir de refuge. De plus, associée à l'extase et à la jouissance, elle apparaît comme synonymique de l'ivresse car promesse de libération et d'exaltation. Au creux et au cœur de la sexualité, "forme laïcisée de l'extase » ${ }^{56}$,

54 M. Belhaj Kacem, «Instinct », op. cit.

55 M. Foessel, La Nuit. Vivre sans témoin, op. cit., p. 60.

56 M. Belhaj Kacem, «Instinct », op. cit. 
s'entrechoquent les deux sens contradictoires de sacer: I'extase divine se confond avec l'orgasme corporel. De la même façon, dans la boîte de nuit, l'orgasme devient eargasm; ce néologisme, qui a pour fonction de désigner l'extase qui peut s'emparer d'un corps à l'écoute d'une musique, illustre la façon dont la musique, intangible, parvient pourtant à s'introduire dans un organisme au point de le bouleverser. Tout porte à croire que la nuit est synonyme d'intensification, intensification qui s'oppose au calme du jour.

Ce qui s'écrit aspire à la puissance de ce qui se sent la nuit, temps où les sensations atteignent leur acmé et où les normes diurnes s'annulent; le paroxysme et l'oxymore apparaissent alors comme les figures fétiches de cette écriture insomniaque, échappant tous deux à la classification précise et raisonnée caractéristique du jour. Les sens perdus dans la nuit s'expriment par ces figures de rhétorique capables de susciter sentiment de vertige et foudroiement d'intensité.

\section{Conclusion}

"Instinct » utilise le terme $d^{\prime}$ '« expérience intérieure ${ }^{57}$ de la nuit à propos des prostituées de White Noise ; on peut également l'utiliser pour 
caractériser la démarche des auteurs de La Nuit Épuisée. La notion d'expérience intérieure fait référence à l'ouvrage de Georges Bataille qui en porte le titre, et c'est ainsi qu'il la définit : « J'entends par expérience intérieure ce que d'habitude on nomme expérience mystique : les états d'extase, de ravissement, au moins d'émotion méditée ${ }^{58}$. L'expérience est par ailleurs pour Bataille « la mise en question (à l'épreuve), dans la fièvre et dans l'angoisse, de ce qu'un homme sait du fait d'être $»{ }^{59}$.

La Nuit Épuisée nous donne à lire douze expériences intérieures de la nuit, douze états d'extase, douze émotions méditées et transcrites dans la fièvre de la nuit. La trame commune de cette polyphonie nocturne est la volonté de s'appuyer sur une expérience intérieure qui soit un bouleversement perceptif, sensitif et émotionnel pour écrire. La poïétique de l'insomnie lisible dans cet ouvrage naît donc de l'épuisement des corps et du dérèglement des sens, tous deux inhérents à la nuit blanche.

Si ces auteurs sont bien porteurs de «paroles incandescentes ${ }^{60}$ qui ont brûlé au cœur de leur

58G. Bataille, L'Expérience intérieure, Paris, Gallimard, 1954, p. 15. $59 \mathrm{lbidem}$.

60 J. Jefferson Selve, « Sacrifice », op. cit. 
insomnie collective, sans doute chacun d'entre eux mérite-t-il le titre de feu follet, « figure transgressive [...] qui danse et court partout dans la nuit en nous affolant de désirs $»{ }^{61}$, feu follet symbolisant les « surgissements vitaux » caractéristiques de « [l']art des extases » ${ }^{62}$ et de « l'art intégral [qui] se réalise dans le chaos de la jouissance et de la fête, dans le renversement de la logique du rendement, dans la célébration païenne de la destruction ${ }^{63}$.

Date de réception de l'article: 15.03.2021 Date d'acceptation de l'article: 11.04.2021

61 G. Didi-Huberman, «Préface », [dans :] G. Bataille, Courts Écrits sur l'art, Paris, Éditions Lignes, 2017, p. 18. 62 lbidem, p. 17-18.

63 A. d'Agata, M. Belhaj Kacem, Stasis. Géométrie de la cruauté, Marseille, Studio Vortex, 2019, page non numérotée. 


\section{bibliographie}

Agata A. d', Garido R., Agonie, Paris, Actes Sud, 2009.

Agata A. d', et al., La Nuit Épuisée, Marseille, Friche la Belle de Mai / André Frère Éditions, 2018.

Agata A. d', Manifeste, Paris, Le Bleu du ciel, 2005.

Agata A. d', Belhaj Kacem, M., Stasis. Géométrie de la cruauté, Marseille, Studio Vortex, 2019.

Bataille G., Courts Écrits sur l'art, Paris, Éditions Lignes, 2017.

Bataille G., La Part Maudite suivie de La Notion de dépense, Paris, Minuit, 1967.

Bataille G., L'expérience intérieure, Paris, Gallimard, 1954.

Debord G., Internationale Situationniste, Juin 1958, $\mathrm{n}^{\circ} 1$. http://hipermedula.org/wp-content/uploads/2013/09/ Internationale_situationniste_1.pdf.

Deleuze G., Critique et Clinique, Paris, Minuit, 1993.

Foessel M., La Nuit. Vivre sans témoin, Paris, Autrement, 2017. Lyotard C., Masson C., "La peinture ambivalente », [dans :] G. Gassiot-Talabot et al., Figurations : 1960/1973, Paris, UGE, 1973.

Merleau-Ponty M., Phénoménologie de la perception, Paris, Gallimard, 1945.

Passeron R., Pour une philosophie de la création, Paris, Klincksieck, 1989.

Savaunet P., « Nietzsche, philosophe-musicien de l'éternel retour », [dans :] Archives de Philosophie, 2001, n² 2001/2, t. 64. Sibertin-Blanc G., « Cartographie et territoire. La spatialité géographique comme analyseur des formes de subjectivité selon Gilles Deleuze », [dans :] L'Espace Géographique, 2010, n²010/3, t. 39. 


\section{abstract}

\section{La Nuit Épuisée, poietic of a collective insomnia}

La Nuit Épuisée (2018), project on the initiative of Antoine d'Agata and Lea Bismuth, comes from a collective writing experience which tooks place during a sleepless night; consequently, it allows us to see the visual and textual marks left by the night on this photoliteracy silkscreened book. By analysing this work and each of his twelve leporelli, we can, like its authors, see what it's only perceptible into the night. As much as poietic is, according to René Passeron, the science of the dynamic relationship between an artist and its artwork while he is struggling with it, this article analyses the context of creation of this book (a collective insomnia in a nightclub) and how it affects the plasticity and the writing of a book which intends to propose us a reading experience.

\section{keywords}

photoliteracy, Antoine d'Agata, insomnia, leporello, La Nuit Épuisée

\section{mots-clés}

photolittérature, Antoine d'Agata, nuit blanche, leporello, La Nuit Épuisée 


\section{ninia bigot}

Nina Bigot est doctorante à l'université de Lille. Sa thèse, dirigée par Florence de Chalonge, s'intitule L'art photolittéraire d'Antoine d'Agata. Ses premières communications et publications portent sur l'esthétique de l'obscène et la politique de l'immonde (comme son intervention pour le colloque international "Politiques et récits du corps ») et l'analyse du scandale dans la photolittérature viatique d'Agata.

ORCID : 0000-0001-9169-9686 